Review Article

\title{
The Clinical Application of Raman Spectroscopy for Breast Cancer Detection
}

\author{
Pin Gao, ${ }^{1}$ Bing Han, ${ }^{1}$ Ye Du, ${ }^{1}$ Gang Zhao, ${ }^{1}$ Zhigang Yu, ${ }^{2}$ Weiqing Xu, ${ }^{3}$ Chao Zheng, ${ }^{2,4}$ and \\ Zhimin Fan $^{1}$
}

${ }^{1}$ Department of Breast Surgery, The First Hospital of Jilin University, Changchun 130021, China

${ }^{2}$ Department of Breast Surgery, The Second Hospital of Shandong University, Jinan 250033, China

${ }^{3}$ State Key Laboratory for Supramolecular Structure and Materials, Jilin University, Changchun 130012, China

${ }^{4}$ Department of Mechanical Engineering, Johns Hopkins University, Baltimore, MD 21218, USA

Correspondence should be addressed to Chao Zheng; czheng5@jhu.edu and Zhimin Fan; fanzhimn@163.com

Received 27 November 2016; Revised 7 February 2017; Accepted 16 February 2017; Published 15 May 2017

Academic Editor: Christoph Krafft

Copyright (C) 2017 Pin Gao et al. This is an open access article distributed under the Creative Commons Attribution License, which permits unrestricted use, distribution, and reproduction in any medium, provided the original work is properly cited.

\begin{abstract}
Raman spectroscopy has been widely used as an important clinical tool for real-time in vivo cancer diagnosis. Raman information can be obtained from whole organisms and tissues, at the cellular level and at the biomolecular level. The aim of this paper is to review the newest developments of Raman spectroscopy in the field of breast cancer diagnosis and treatment. Raman spectroscopy can distinguish malignant tissues from noncancerous/normal tissues and can assess tumor margins or sentinel lymph nodes during an operation. At the cellular level, Raman spectra can be used to monitor the intracellular processes occurring in blood circulation. At the biomolecular level, surface-enhanced Raman spectroscopy techniques may help detect the biomarker on the tumor surface as well as evaluate the efficacy of anticancer drugs. Furthermore, Raman images reveal an inhomogeneous distribution of different compounds, especially proteins, lipids, microcalcifications, and their metabolic products, in cancerous breast tissues. Information about these compounds may further our understanding of the mechanisms of breast cancer.
\end{abstract}

\section{Introduction}

Breast cancer is the most common cancer in women worldwide. In 2012, nearly 1.7 million people were diagnosed with breast cancer [1]. Reducing the incidence and death of breast cancer is a major public health priority. Once a mass is found in the breast, ultrasonography, diagnostic mammography, and/or magnetic resonance imaging (MRI) will be recommended by a physician. If the mass is possibly malignant, a core needle biopsy and histopathological techniques are necessary [2]. Unfortunately, this invasive examination cannot ensure a $100 \%$ correct diagnosis. If the lesion is malignant or the possibility of malignancy cannot be excluded, the patient will undergo surgery. Surgery for breast cancer includes lesion resection and axillary lymph node resection. In breast surgery, breast-conserving surgery and sentinel lymph node biopsy (SLNB) are two breakthroughs. SLNB results in less lymphedema of the upper arm [3]. Both techniques require an intraoperative pathological diagnosis, which usually takes at least $30 \mathrm{~min}$. After mastectomy for breast cancer, some patients also need chemotherapy, radiotherapy, and endocrine therapy. The necessity of molecular-targeted therapy is based on the result of fluorescence in situ hybridization (FISH). After operation, [(18)F]-2-fluoro-2-deoxy-D-glucose positron emission tomography/computed tomography (FDG$\mathrm{PET} / \mathrm{CT})$ can be used for detecting the recurrence and metastasis [4]. Despite advances in the diagnosis and treatment of breast cancer, some limitations remain:

(1) Neither ultrasound nor mammography can make a qualitative diagnosis, so invasive biopsy or even surgery remains the gold standard in breast cancer.

(2) Sentinel lymph nodes in intraoperative frozen sections cannot identify the metastasis of the lymph node with $100 \%$ accuracy [5]. 
(3) After the lesion is removed, there is no way to evaluate the effect of chemotherapy or molecular-targeted therapy.

(4) Examinations such as pathology in current clinical application cannot provide information at the molecular or cellular level.

This review introduces some of the latest progress of Raman spectroscopy, a promising technique in breast cancer diagnosis. This optical technique has the potential to solve the problems listed above. First, Raman spectra can provide information of molecules (e.g., lipids, DNA, and proteins) to distinguish cancerous lesions from noncancerous lesions. Molecular changes may also indicate the mechanism of cancer development. Due to its real-time characteristics, Raman spectroscopy is a powerful intraoperative diagnostic technique. Furthermore, when combined with a nanotag, Raman spectroscopy can reveal whether cancer is sensitive or resistant to an anticancer drug.

\section{Brief Overview of Raman Spectroscopy}

When a photon interacts with a molecule, elastic or inelastic collision happens. During inelastic collision, there is an exchange of energy between the photon and the molecule. The photon changes its motion direction after the collision (Figure 1). Meanwhile, the photon passes part of the energy to the molecule or obtains energy from the molecule to change the photon's frequency. This process is called Raman scattering. In this process, if the incident photons lose energy, the scattered photons have a lower frequency. In contrast, if the incident photons get more energy from the environment, the scattered photons have a higher frequency. If the difference of the energy between the incident and scattered photons is monitored, information on the frequency and vibration can be obtained and used to analyze the sample compounds [6]. After Raman spectral imaging is obtained, mathematical analyses are necessary to extract the useful information from the test. Many spectral unmixing algorithms are applied currently, including principal component analysis (PCA), vertex component analysis (VCA), hierarchical cluster analysis (HCA), support vector machine (SVM), and SVM-recursive feature elimination (SVM-RFE) [7]. PCA is more commonly used for quantitative analysis in detecting cancers such as melanoma, breast cancer, and cervical cancer [8-10]. As for HCA, it is used for cancer diagnosis such as esophageal cancer [11] and some cancer cells [12-14]. Besides, SVM-RFE can be used to analyze and identify the most distinctive spectral features and, thus, has higher sensitivity and specificity [7].

The advantages of Raman spectroscopy include almost all those of optical detections. Raman spectroscopy has high resolution; it is safe, noninvasive, and nonradiative; and it can continuously monitor soft tissues. Compared with fluorescence spectrum, the Raman spectrum can reflect the molecular structures and biochemical compositions without the need to process samples in advance [15]. Although Raman spectroscopy offers highly specific information on

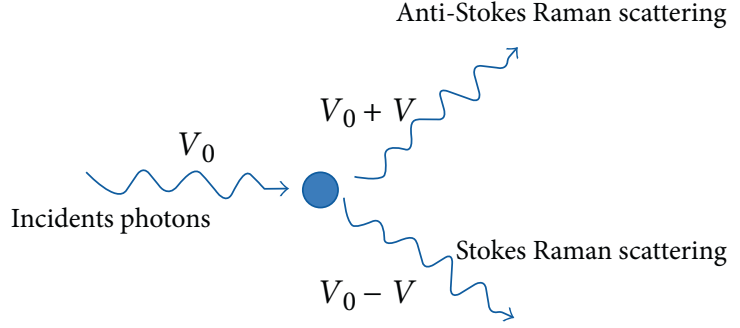

FIgURE 1: Schematic of inelastic collision showing anti-Stokes Raman scattering and Stokes Raman scattering.

the molecular features of tissues, its use has remained limited because of its relatively low ability to produce distinguishable signals. However, conventional Raman signals are less sensitive than fluorescence. To solve this problem, surfaceenhanced Raman spectroscopy (SERS) was developed. When some nanoparticles absorbed to the surface of some rough metal, such as gold and silver, the Raman signal is amplified. This phenomenon is called SERS. One of the most important mechanisms of SERS is the enhancement of the electromagnetic field through the localization of optical fields in metallic nanostructures [16]. Although SERS can amplify Raman signals, it has some limitations [6]. First, the colloidal aggregation added to samples has not been proven able to amplify Raman signals. Second, there may be side reactions in the process. The spectra of the sample and nanoparticle may overlap. Third, when the nanoparticle is added to the analyte, there may be more toxic reactions. Despite these limitations, SERS remains a very important research direction of Raman spectroscopy.

In clinical application, Raman spectroscopy has been a rapidly developed technology that monitors signals at different levels. At the molecular level, it can be used to analyze DNA sequences and gene products. At the cellular level, Raman spectra can monitor the process of malignant cells or relative intercellular processes in the peripheral blood of cancer patients [6]. The information can then be used to evaluate cancer cell metabolism, proliferation, and even metastasis. Furthermore, Raman spectroscopy provides a promising way to detect biomarkers that are expressed on the surfaces of tumor cells $[17,18]$. All of this information can be used to help doctors to diagnose diseases, determine the appropriate treatment, and even evaluate prognosis. To date, Raman technology has been used to diagnose many cancers, including cervical cancer, malignant skin tumors, gastric cancer, colorectal cancer, and breast cancer [19-22]. This article aims to review the application of Raman spectra in breast cancer and describe the newest progress in this field.

\section{Application of Raman Spectroscopy in Breast Cancer}

Raman spectroscopy is noninvasive and has high specificity, making its clinical application very promising. Nowadays, studies focus on using Raman spectroscopy to differentiate between benign and malignant tissues in place of traditional biopsy [23]. Raman spectroscopy can provide realtime information, making its intraoperative use possible. 
Monitoring sentinel lymph node and surgical margins for breast-conserving surgery using Raman spectra can shorten the operative time. If focusing on a single cell, it no longer requires several days to detect biomarkers on the cell surface as with FISH. To determine the efficacy of anticancer drugs, Raman spectra are used to monitor cancer cell metabolism and composition. This is a new research direction that aims to elucidate how DNA, protein, lipid, and calcification contribute to cell proliferation in vivo that will further our understanding of the pathogenesis of cancer.

3.1. Raman Spectroscopy in Tissues. Generally, Raman bands associate with the vibration of a special chemical bond or a distinct functional group in the molecule. Therefore, based on distinctive spectral features and intensity differences, we could discriminate normal, benign, and malignant. In recent years, many research papers have also shown the availability of Raman spectroscopy to classify diseased tissue in the breast $[7,24]$ and especially for intraoperative margin assessment and SLNB for breast cancer.

\subsubsection{Qualitative Diagnosis of Breast Disease with Raman} Spectroscopy. Many studies have shown that the spectral profiles of normal breast (NB) tissue peaks appear at 1004, 1080, $1158,1266,1304,1444,1518,1660,1750,2674,2727,2854$, 2888,2926 , and $3009 \mathrm{~cm}^{-1}$, while those of malignant tissue peaks appear at 1004, 1259, 1444, 1660, 2854, 2888, 2926, 3009 , and $3311 \mathrm{~cm}^{-1}$. In normal tissues, the peaks at 1004 , 1158 , and $1518 \mathrm{~cm}^{-1}$ belong to carotenoids $(\mathrm{C}-\mathrm{C}, \mathrm{C}=\mathrm{C}$ ), whereas in malignant tissues, the peaks at 1080, 1259, 1444, 1660,2854 , and $2940 \mathrm{~cm}^{-1}$ are more obvious. Peaks at $2854 \mathrm{~cm}^{-1}$ belong to lipids, while those at $2940 \mathrm{~cm}^{-1}$ are believed to represent mixtures of lipids and proteins [26]. Shell-isolated nanoparticle-enhanced Raman spectroscopy (SHINERS) is a powerful SERS substrate. After SHINERS is added to a sample, Raman spectral intensity is amplified. Knowing the magnification of different peaks is helpful in diagnosing breast diseases. Researchers have also utilized the Au@SiO2 SHINERS technique to detect NB tissues, fibroadenoma (FD), atypical ductal hyperplasia (ADH), ductal carcinoma in situ (DCIS), and invasive ductal carcinoma (IDC). Compared with traditional Raman spectroscopy, SHINERS can provide more spectral feature information (Figure 2) [24, 25].

Studies of cells in response to extracellular signals using two-dimensional (2D) culture conditions, including cultures of isolated, cloned, and immortal cells that grow on rigid surfaces, date back to the 1900s. The limitation of this type of model is that it does not consider the extracellular matrix (ECM), a very important factor in cell phenotypes [27]. In 1977, Emerman and Pitelka found that a deformable threedimensional ECM (3D-ECM) environment is necessary for maintaining the tissue structure of NB tissue [28]. Because 3D-ECM cultures can better mimic in vivo environments, Damayanti et al. first combined 3D-ECM cultures with Raman spectroscopy to study breast cancer [29]. In the 3DECM culture model, peaks of precancerous lesions were observed at 1451 and $1650 \mathrm{~cm}^{-1}$, while peaks of noncancerous tissues were observed at $1650 \mathrm{~cm}^{-1}$. Different from traditional Raman spectroscopy, the signal intensity in precancerous lesions is stronger than that in noncancerous tissues. In addition, the sensitivity and specificity are higher in 3D-ECM cultures than in 2D cultures. Microenvironment is a major factor in modulating cell morphology and proliferation [30]. Changes in the surrounding stroma may be an initial factor of precancerosis.

3.1.2. Intraoperative Raman Spectroscopy. Considering its real-time nature without the need for preparation, Raman spectroscopy is a promising new technique in intraoperative margin assessment and SLNB.

(1) Raman Spectroscopy of Surgical Margins. Haka and colleagues first used Raman spectra to examine surgical margins in nine patients during partial mastectomy surgeries [31]. Compared with pathological examinations after surgeries, the algorithm results in an overall accuracy of $93.3 \%$. Conventional Raman spectroscopy has a depth limitation. Spatially offset Raman spectroscopy (SORS) is a new technique that was first applied by Matthew for cancer diagnosis. SORS can detect deeper tissues at least $0.5-2 \mathrm{~mm}$ below the surface [32]. The use of SORS in intraoperative margin assessment has $95 \%$ sensitivity and $100 \%$ specificity [33]. In recent years, Mohs et al. developed a handheld spectroscopic pen device (termed SpectroPen) using both a fluorescent contrast agent (indocyanine green) and a SERS contrast agent (pegylated colloidal gold). The team gained real-time information on tumor margins. In that study, the tissue penetration depth was $5-10 \mathrm{~mm}$ depending on the tissue's optical properties and the ability to resolve contrast agent signals [34]. They also constructed a fiber optic spectroscopic system with stable alignment. The SpectroPen has also been used to detect bioluminescent $4 \mathrm{~T} 1$ breast tumors of in vivo mouse models. In the intraoperative measurement, the SpectroPen is helpful for distinguishing tumor margins and detecting whether any tumor remains.

(2) Raman Spectroscopy in SLNB. Another important application of Raman spectra is in SLNB. In traditional operations, surgeons use dyes or radionuclides Te-99 to localize sentinel lymph nodes. However, the rates of localizing sentinel lymph nodes through these methods are $73.8 \%$ and $94.1 \%$, respectively [35]. After lymph node removal, surgeons must wait for the immunohistochemical analysis (IHA) results to decide if axillary lymph node dissection is necessary, which usually takes $30-60 \mathrm{~min}$. In some cases, a second operation cannot be avoided because the specificity of frozen section rapid pathologic examination cannot reach $100 \%$ and the false negative rate is at least $4 \%$ [36]. Isabelle et al. first used Raman spectra and infrared images to detect the lymph node excision during head and neck tumor surgeries [37] and showed that lymph nodes with metastatic cancer have greater nucleic acid and less lipid and carbohydrate content than normal lymph nodes. These results suggest that lymph nodes with metastatic cancer grow faster and are much more poorly differentiated than normal lymph nodes.

Smith et al. used mapping Raman spectra to accurately judge lymph node metastases [38]. Although metastatic 

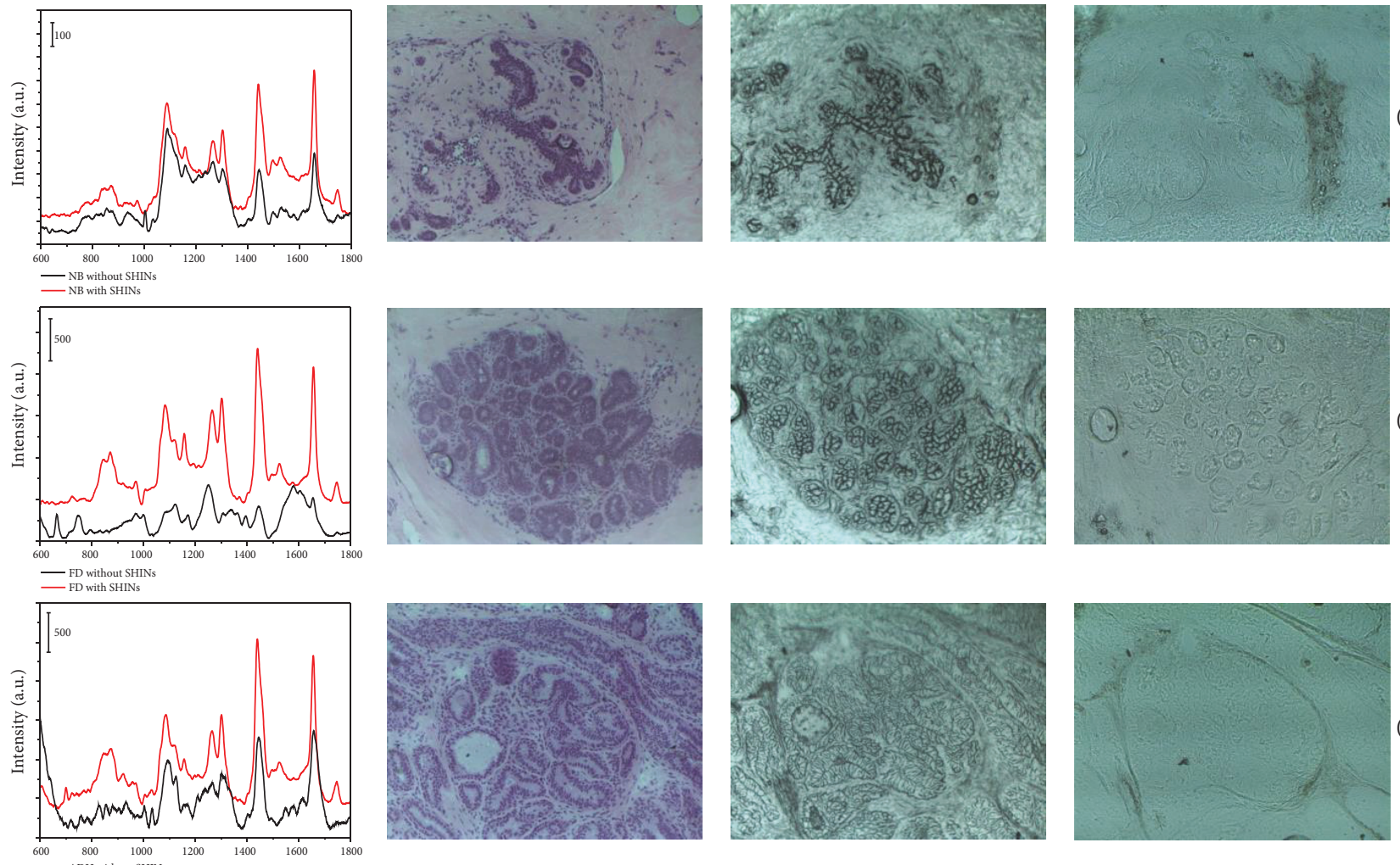

$(\mathrm{ADH})$
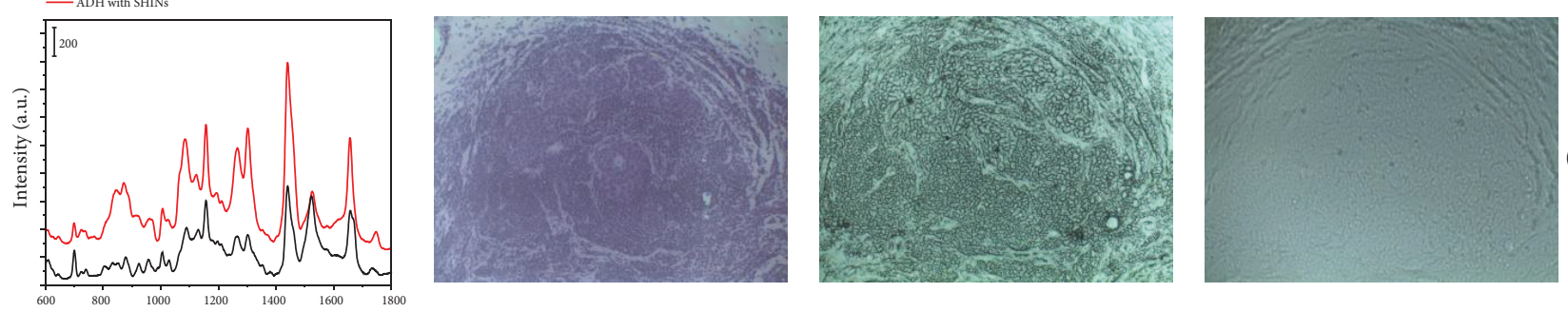

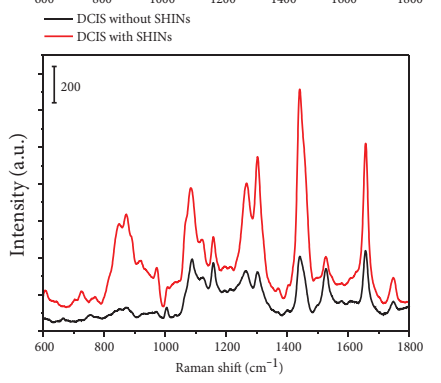
二 ${ }^{\text {IDC without SHINs }}$

(a)

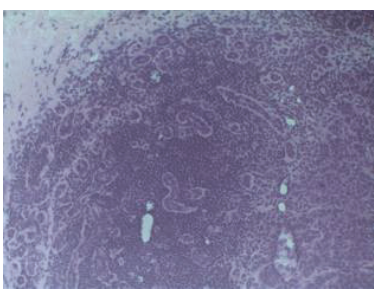

(b)

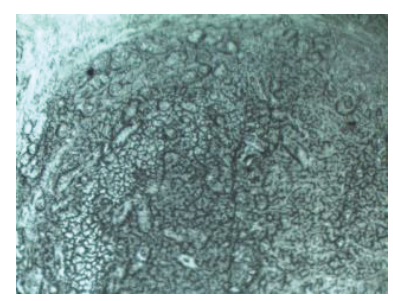

(c)

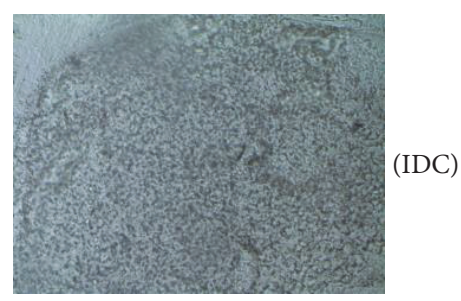

(d)

FIGURE 2: Diagnosis of breast cancer with Raman spectroscopy. (a) Mean Raman spectra and SHINERS spectra of breast tissues: normal breast (NB) tissues, fibroadenoma (FD), atypical ductal hyperplasia (ADH), ductal carcinoma in situ (DCIS), and invasive ductal carcinoma (IDC). (b) Corresponding images of frozen sections were stained with HE. (c) Corresponding images of frozen sections without shell-isolated nanoparticles (SHINs). (d) Corresponding images of frozen sections with SHINs. The images show an area of $0.8 \times 0.6 \mathrm{~mm}^{2}$ [24, 25].

cancer is successfully diagnosed, the running time of each section is $12-120$ hours. Such a long running time is not suitable for real-time tissue diagnosis. To overcome this limitation, Horsnell et al. developed a method of recording only five spectra of each lymph node [39]. The spectra gained from 38 lymph nodes of 20 patients indicated that the fatty acid, collagen, and protein contents differ between metastatic and normal lymph nodes. Among the four standards in the study, if a node was classified based on the simple majority of the five random spectra, the sensitivity and specificity were higher than those in the other groups. Thereafter, to study the effect of the number of spectra, Horsnell et al. compared five- and 10-point probe analyses [40]. The assessment of the lymph node based on spectra from five points achieved $71 \%$ 
sensitivity and $97 \%$ specificity, while the 10 -point method achieved $81 \%$ sensitivity and $97 \%$ specificity. The sensitivity and specificity of 10-point spectra are comparable to those of other current methods of intraoperative assessment. The running time in these methods are 9 and $18 \mathrm{~min}$, respectively, much shorter than the current methods, making Raman spectra a very promising technique in the diagnosis of lymph node metastasis and more suitable for clinical application than the currently used techniques.

\subsection{Raman Spectroscopy in Cells}

3.2.1. Raman Spectroscopy in Single Cells and the Cellular Microenvironment. Neugebauer et al. identified single cells in the circulation using Raman spectra [41]. Isolating leukocytes and erythrocytes from breast carcinoma-derived cells (MCF-7, BT-20) can achieve a sensitivity $>99.7 \%$ and a specificity $>99.5 \%$. The team used SVMs with a linear kernel to develop a classification model. Moreover, using Ramanactivated cell sorting, Dochow et al. successfully identified leucocytes extracted from blood, BT-20 and MCF-7 breast cancer cells, and OCI-AML3 leukemia cells [42, 43].

Many genetic and environmental factors contribute to the development of breast cancer. An enhanced understanding of these factors can provide more information on the pathogenesis of cancer and guide its treatment. Therefore, using various biomarkers and other testing methods to study tumor-associated molecules is a promising research direction. Raman is a good option because it can test unlabeled lesion as well as nucleic acid and surface receptor products using specific probes [6].

\subsubsection{Efficacy of Chemotherapy in Breast Cancer with Raman} Spectroscopy. Raman spectroscopy has been used to increase the efficacy of chemotherapy in breast cancer, to evaluate the resistance of chemotherapy, and to monitor the damage of cells due to chemotherapy. Hossain et al. first used SERS and a kind of biohybrid nanoparticles to increase the doxorubicin delivery. Meanwhile, this system also helped monitor the metabolism of drugs in breast cancer cells [44]. Lapatinib is another important oral anticancer drug with low absorption. Considering its high-binding ability with albumin, human serum albumin nanoparticles loaded with lapatinib obviously increased the efficacy of the drug [45]. Hu et al. designed a novel type of nanomedical platform with double-walled $\mathrm{Au}$ nanocage/ $\mathrm{SiO}(2)$ nanorattle and improved biocompatibility as well as cell uptake ability, which in turn increased the efficacy of chemotherapy [46]. The human epidermal growth factor receptor 2 (HER2) proto-oncogene is amplified in $20-30 \%$ of human breast cancer cases. Studies have shown that patients with HER2-positive breast cancer are at increased risks of recurrence and metastasis [47]. HER2 proto-oncogene expression can be detected by Raman spectra, which have also been used to detect HER2 expression in the BT474 (HER2+), MCF-10A (HER2-), and MCF-10A (HER2+) cell lines [48]. In this study, the application of Raman spectra was first used to characterize the biochemical response of drug resistance and sensitivity to lapatinib, a targeted antitumor agent. After long time of chemotherapy, cells would be damaged in vivo. Ilkhani et al. designed a novel biosensor to assess the damage of DNA after chemotherapy with the help of SERS [49].

\subsection{Composition Analysis of Samples with Raman} Spectroscopy. Raman spectra can exhibit spectral features including composition and structure changes such as peptide conformation, biomarkers, DNA sequences, base composition, microcalcifications, and lipids.

3.3.1. Raman Spectroscopy of Proteins. As a fast technique with high specificity, Raman spectra can also analyze proteins of cancer in a low-sample volume, especially in the detection of cell surface biomarkers. Current techniques such as enzyme-linked immunosorbent assays (ELISA) usually detect a sample with a concentration in the nanograms-tograms per milliliter range. However, at the early cancer stage, the cancer proteins are at a low concentration that may be below the ELISA limit of detection. Dinish et al. developed a new hollow core photonic crystal fiber to detect low amounts of proteins [50]. It is well known that epidermal growth factor receptor (EGFR) is a very important biomarker in breast cancer. In that study, EGFR antibody is combined with SERS nanotags. Through this method, Raman spectra can detect proteins at a concentration in the range of $\sim 100 \mathrm{pg}$ in the volume of $\sim 10 \mathrm{~nL}$. Nilstad et al. also used SERS to detect epidermal growth factor receptor (EGFR) expression and investigate the therapeutic effects of cetuximab antibodies [51].

Besides EGFR, Lee et al. [52] used silica-encapsulated hollow gold nanospheres (SEHGNs) as a Raman nanotag that is conjugated with specific antibodies. This technique enables the assessment of the expression of epidermal growth factor (EGF), HER2, and insulin-like growth factor-1 receptors in the MDA-MB-468, KPL4, and SK-BR-3 human breast cancer cell lines. That study indicated that SEHGNs may possibly be used in the early diagnosis of breast cancer.

HER2 proto-oncogene encodes a protein that has extracellular, transmembrane, and intracellular domains that are consistent with the structures of a growth factor receptor. Raman spectra have shown a prominent presence of lipid in the MDA-MB-231 (HER2-negative), MDA-MB-435s (HER2-positive), and SK-BR-3 (HER2 overexpressed) cell lines [13]. Different from current tests, the application of SERS in tumor cell surface biomarkers such as HER2 is more effective than that of IHA and FISH.

Another successful application of SERS is the singlemolecule detection of BRCA1 by Wabuyele et al. [53]. The probe has one DNA sequence complimentary to the target sequence and two arms, one that is connected with a SERSactive molecule and the other that is connected with a metallic nanoparticle. The metallic nanoparticle enhances the SERS signal. The probe looks like a hair loop without the target sequence and maintains a Raman label. Once the BRCA1 gene mutation occurs, the mutated target sequence combines with the probe, the Raman label separates from the metal nanoparticle, and the signal decreases.

A new simultaneous SERS method was developed by Li et al. [15] which consists of a novel Raman probe of branched DNA-gold nanoaggregates that can simultaneously monitor 


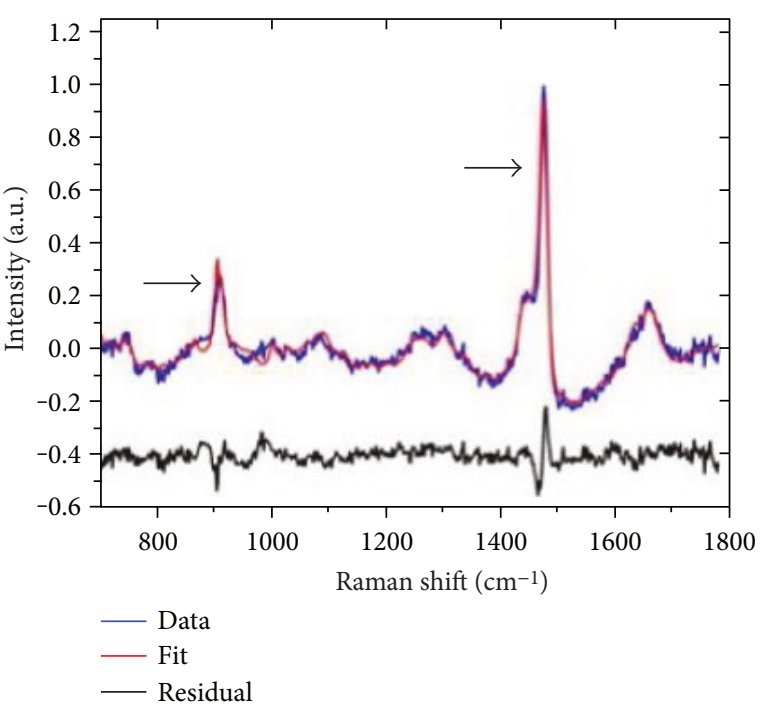

(a)

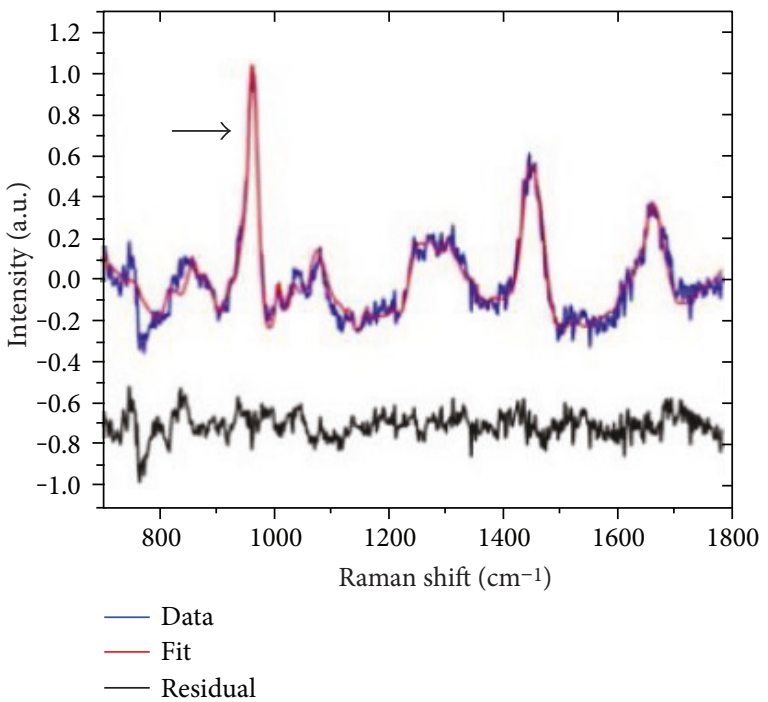

(c)

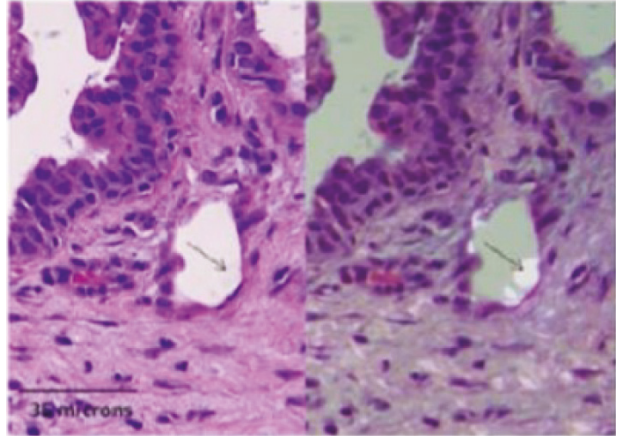

(b)

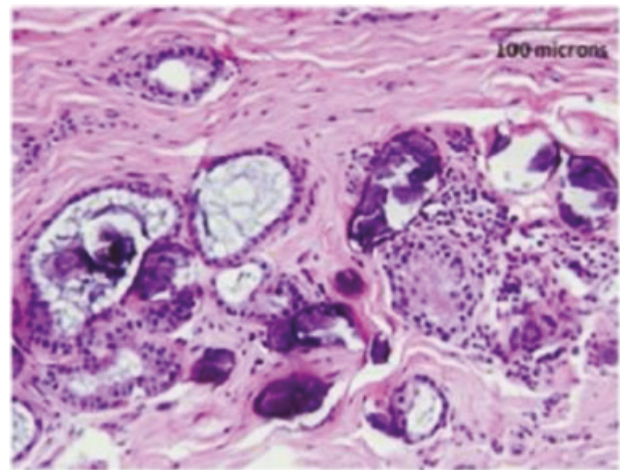

(d)

FIgURe 3: Typical Raman spectra and histopathology of breast lesions (fibrocystic change) with type I and II microcalcifications. The Raman spectrum of the breast lesion with type I microcalcifications in (a) shows prominent bands at $912 \mathrm{~cm}^{-1}$ and $1477 \mathrm{~cm}^{-1}$ (arrows) characteristic of calcium oxalate; the calcium oxalate crystals comprising the type I microcalcifications (b) do not bind $\mathrm{H} \& \mathrm{E}$ (left panel) and appear as colorless crystals (arrows) that are birefringent when viewed under polarized light (right panel). In contrast, the Raman spectrum of the breast lesion with type II microcalcifications in (c) shows a prominent band at $960 \mathrm{~cm}^{-1}$ (arrow) characteristic of calcium hydroxyapatite; the calcium hydroxyapatite rich type II microcalcifications appear as basophilic concretions on the H\&E stain (d) and are nonbirefringent $[59,60]$.

two MCF-7 cell line markers. This development makes Raman spectroscopy more effective for clinical diagnosis. Using an optoelectrofluidic device and a liquid crystal display module, Hwang et al. successfully detected another human tumor marker, $\alpha$-fetoprotein, in a sample volume of $\sim 500 \mathrm{~nL}$ [54]. Combined with fluorescence dual-modal nanoprobes (DMNPs), Lee et al. also got the imaging of CD24 and CD44 expressed on the surface of breast cancer cells [55]. Kang et al. used SERS with channel-compressed spectrometry to detect EGFR, HER2, CD24, and CD44 in fresh tissue specimens with less errors and lower concentrations [56].
This simple, fast, and highly sensitive automated technique opens a new method of clinical diagnosis.

3.3.2. Raman Spectroscopy of Microcalcifications. Breast microcalcifications are key features for the diagnosis of malignant tumors and sometimes the only feature of mammography. In many clinical cases, calcification is a poor prognostic factor for patients with IDC. Mammography is currently the most effective technique to detect calcifications in clinical application despite its limitation of not distinguishing malignant from benign lesions. Mammographic 
images can show the morphological characteristics of different calcifications and help predict the tumor characteristics. Only after stereotactic vacuum-assisted breast biopsies and subsequent pathological diagnosis [57] can doctors make an accurate diagnosis of a lesion. However, this kind of invasive medical examination may fail to retrieve lesions with microcalcifications in about $15 \%$ of all cases. Follow-ups have shown that the cancer diagnosis is missed in $8 \%$ of patients [58]. Therefore, a new noninvasive technique that can gain information on microcalcifications in real time with higher sensitivity and specificity is urgently needed.

There are two types of microcalcifications in breast tumors (Figure 3) [59-61]. Type I microcalcifications, which consist of calcium oxalate, are mainly associated with benign breast disease, whereas type II microcalcifications, which consist of carbonated calcium hydroxyapatite, can be found in proliferative breast lesions, including invasive and in situ cancer. Raman spectroscopy can distinguish the two types of microcalcifications in breast diseases [62] and thus distinguish malignant from benign lesions. In type II microcalcifications, there is more carbonate in benign disease such as sclerosing adenosis than in malignant lesions, although malignant lesions usually contain greater amounts of proteins, amino acid residues, and carotenoids. Baker et al. observed a significant correlation between carbonate concentrations and carcinoma in situ subgrades [63]. In a recent study, an Au@SiO2 SHINERS technique was utilized in frozen sections of surgical resection or Mammotome biopsy. The SHINERS spectra can probe the presence of calcified deposits and distinguish among normal breast tissues, fibroadenoma, atypical ductal hyperplasia, ductal carcinoma in situ (DCIS), and invasive ductal carcinoma (IDC). By correlating the spectra with the corresponding histologic assessment, the study developed partial least squares discriminant analysisderived decision algorithm that provides excellent diagnostic power in the fresh frozen sections (overall accuracy of $99.4 \%$ and $93.6 \%$ using SHINs for breast lesions with and without microcalcifications, resp.) $[24,64]$.

3.3.3. Raman Spectroscopy of Lipids and Other Metabolites. In addition to microcalcifications, there are significant differences in vibrational spectroscopy of proteins, lipids, and carotenoids between benign and malignant lesions, especially unsaturated fatty acids. It is well known that lipids are crucial in biological membranes. If abnormal metabolism occurs in the local enzymes responsible for lipid synthesis and degradation that results in alterations in the expression of genes encoding these enzymes, lipids may change and affect the membranes [26]. Another important factor of cell stability is carotenoid, which can neutralize reactive oxygen species and can reduce oxidative DNA damage and genetic mutations. Carotenoids can act as a protective factor against breast cancer [26, 65]. Raman spectroscopy can differentiate noncancerous from cancerous tissues through carotenoid and lipid levels [17]. Hartsuiker et al. reported variance in lipid levels among breast cancer cell lines with varying HER2 expression levels [13]. Bi et al. used Raman spectroscopy to detect lipids in cell lines [48]. In the cell lines treated with lapatinib, a drug-targeting HER2, the lipid levels were increased in the drug-resistant cells. These results suggest a correlation between lipids and drug resistance.

Progestogen is a potent factor in the proliferation of breast carcinoma cells [66]. Potcoava et al. exposed T47D breast carcinoma cells to medroxyprogesterone acetate and the synthetic androgen R1881 [67]. Raman spectral imaging showed the process of hormone-mediated lipogenesis. Hormone-treated T47D cancer cells proliferate faster than untreated cell. Hedegaard et al. have first used Raman spectra to demonstrate the difference between metastatic cell lines and nonmetastatic cell lines through the content of unsaturated fatty acid [68]. Hartsuiker et al. evaluated lipid contents in two cell lines: SK-BR-3, with low metastatic potential, and MDA-MB, which are highly metastatic [13]. Raman imaging demonstrated that the lipid contents of MDA-MB cell lines are higher than those of SK-BR-3 cells, although HER2 expression is much higher in SK-BR-3 cells. These results indicate that lipid contents may be a more potent predictive factor of breast cancer than HER2 expression.

\section{Conclusions}

In this article, we have reviewed the clinical applications of Raman spectroscopy for breast cancer over the past few years. Raman spectroscopy has become a promising technique that can distinguish cancerous tissues from normal ones, combine with anticancer drugs for treatment, and detect components at the molecular level including proteins, lipids, and microcalcifications. The differences in components between cancerous and noncancerous tissues can provide information about cancer pathogenesis. While we are excited that Raman spectroscopy may become a new method of clinical diagnosis in breast cancer, we should note that some problems still need to be solved before it is widely used clinically, such as the stability of its sensitivity and predictability as well as how we can overcome the limitation of SERS to obtain better spectra. More importantly, it is necessary to construct a mathematical model using Raman spectrum data that are obtained from normal and malignant tissues based on various biochemical characteristics such as calcification, protein structure, and fat structure. This algorithm would allow us to identify early pathological changes, to distinguish the nature of breast tissues (normal, benign, or malignant), and to explore the inner link between breast cancer and premalignant lesions. The application of Raman spectroscopy can be an efficient way to reduce the need for unnecessary surgical biopsy and significantly save medical resources. Furthermore, it can create a beneficial economic advantage and decrease the psychological burden of patients.

\section{Conflicts of Interest}

The authors declare that they have no conflict of interest and no competing interest. The authors have no other relevant affiliations or financial involvement with any organization or entity with a financial interest in or financial conflict with the subject matter or materials discussed in the manuscript apart from those disclosed. 


\section{Acknowledgments}

This work was supported by the National Natural Science Foundation of China (Grant no. 81202078), the Seed Fund of the Second Hospital of Shandong University, and the National Construction of High Quality University Projects of Graduates from the China Scholarship Council (Grant no. 201406170141).

\section{References}

[1] L. A. Torre, F. Bray, R. L. Siegel, J. Ferlay, J. Lortet-Tieulent, and A. Jemal, "Global cancer statistics, 2012," CA: A Cancer Journal for Clinicians, vol. 65, no. 2, pp. 87-108, 2015.

[2] J. Depciuch, E. Kaznowska, I. Zawlik et al., "Application of Raman spectroscopy and infrared spectroscopy in the identification of breast cancer," Applied Spectroscopy, vol. 70, no. 2, pp. 251-263, 2016.

[3] Y. J. Choi, J. H. Kim, S. J. Nam, Y. H. Ko, and J. H. Yang, "Intraoperative identification of suspicious palpable lymph nodes as an integral part of sentinel node biopsy in patients with breast cancer," Surgery Today, vol. 38, no. 5, pp. 390394, 2008.

[4] N. Y. Jung, R. Yoo Ie, B. J. Kang, S. H. Kim, B. J. Chae, and Y. Y. Seo, "Clinical significance of FDG-PET/CT at the postoperative surveillance in the breast cancer patients," Breast Cancer (Tokyo, Japan), vol. 23, no. 1, pp. 141-148, 2016.

[5] U. Veronesi, G. Paganelli, V. Galimberti et al., "Sentinel-node biopsy to avoid axillary dissection in breast cancer with clinically negative lymph-nodes," Lancet (London, England), vol. 349, no. 9069, pp. 1864-1867, 1997.

[6] H. Abramczyk and B. Brozek-Pluska, "Raman imaging in biochemical and biomedical applications. Diagnosis and treatment of breast cancer," Chemical Reviews, vol. 113, no. 8, pp. 5766-5781, 2013.

[7] C. Hu, J. Wang, C. Zheng et al., "Raman spectra exploring breast tissues: comparison of principal component analysis and support vector machine-recursive feature elimination," Medical Physics, vol. 40, no. 6, p. 063501, 2013.

[8] B. Bodanese, F. L. Silveira, R. A. Zangaro, M. T. Pacheco, C. A. Pasqualucci, and L. Silveira Jr., "Discrimination of basal cell carcinoma and melanoma from normal skin biopsies in vitro through Raman spectroscopy and principal component analysis," Photomedicine and Laser Surgery, vol. 30, no. 7, pp. 381387, 2012.

[9] E. Vargas-Obieta, J. C. Martinez-Espinosa, B. E. Martinez-Zerega, L. F. Jave-Suarez, A. Aguilar-Lemarroy, and J. L. Gonzalez-Solis, "Breast cancer detection based on serum sample surface enhanced Raman spectroscopy," Lasers in Medical Science, vol. 31, no. 7, pp. 1317-1324, 2016.

[10] J. L. Gonzalez-Solis, J. C. Martinez-Espinosa, L. A. TorresGonzalez, A. Aguilar-Lemarroy, L. F. Jave-Suarez, and P. Palomares-Anda, "Cervical cancer detection based on serum sample Raman spectroscopy," Lasers in Medical Science, vol. 29, no. 3, pp. 979-985, 2014.

[11] X. Li, T. Yang, S. Li, D. Wang, and D. Guan, "Detecting esophageal cancer using surface-enhanced Raman spectroscopy (SERS) of serum coupled with hierarchical cluster analysis and principal component analysis," Applied Spectroscopy, vol. 69 , no. 11 , pp. 1334-1341, 2015.
[12] F. Draux, C. Gobinet, J. Sule-Suso et al., "Raman spectral imaging of single cancer cells: probing the impact of sample fixation methods," Analytical and Bioanalytical Chemistry, vol. 397, no. 7, pp. 2727-2737, 2010.

[13] L. Hartsuiker, N. J. Zeijen, L. W. Terstappen, and C. Otto, “A comparison of breast cancer tumor cells with varying expression of the Her2/neu receptor by Raman microspectroscopic imaging," The Analyst, vol. 135, no. 12, pp. 3220-3226, 2010.

[14] T. Tolstik, C. Marquardt, C. Matthaus et al., "Discrimination and classification of liver cancer cells and proliferation states by Raman spectroscopic imaging," The Analyst, vol. 139, no. 22, pp. 6036-6043, 2014.

[15] Y. Li, X. Qi, C. Lei, Q. Yue, and S. Zhang, "Simultaneous SERS detection and imaging of two biomarkers on the cancer cell surface by self-assembly of branched DNA-gold nanoaggregates," Chemical Communications (Cambridge, England), vol. 50, no. 69, pp. 9907-9909, 2014a.

[16] D. Graham and K. Faulds, "Quantitative SERRS for DNA sequence analysis," Chemical Society Reviews, vol. 37, no. 5, pp. 1042-1051, 2008.

[17] H. Abramczyk, B. Brozek-Pluska, J. Surmacki, J. JablonskaGajewicz, and R. Kordek, "Raman 'optical biopsy' of human breast cancer," Progress in Biophysics and Molecular Biology, vol. 108, no. 1-2, pp. 74-81, 2012.

[18] H. N. Wang and T. Vo-Dinh, "Multiplex detection of breast cancer biomarkers using plasmonic molecular sentinel nanoprobes," Nanotechnology, vol. 20, no. 6, p. 065101, 2009.

[19] I. R. Ramos, A. Malkin, and F. M. Lyng, "Current advances in the application of Raman spectroscopy for molecular diagnosis of cervical cancer," BioMed Research International, vol. 2015, p. $561242,2015$.

[20] N. Kourkoumelis, I. Balatsoukas, V. Moulia, A. Elka, G. Gaitanis, and I. D. Bassukas, "Advances in the in vivo Raman spectroscopy of malignant skin tumors using portable instrumentation," International Journal of Molecular Sciences, vol. 16, no. 7, pp. 14554-14570, 2015.

[21] H. H. Kim, "Endoscopic Raman spectroscopy for molecular fingerprinting of gastric cancer: principle to implementation," BioMed Research International, vol. 2015, p. 670121, 2015.

[22] S. Li, G. Chen, Y. Zhang et al., "Identification and characterization of colorectal cancer using Raman spectroscopy and feature selection techniques," Optics Express, vol. 22, no. 21, pp. 25895-25908, 2014b.

[23] A. Saha, I. Barman, N. C. Dingari et al., "Precision of Raman spectroscopy measurements in detection of microcalcifications in breast needle biopsies," Analytical Chemistry, vol. 84, no. 15, pp. 6715-6722, 2012.

[24] C. Zheng, W. Shao, S. K. Paidi et al., "Pursuing shell-isolated nanoparticle-enhanced Raman spectroscopy (SHINERS) for concomitant detection of breast lesions and microcalcifications," Nanoscale, vol. 7, no. 40, pp. 16960-16968, 2015.

[25] C. Zheng, L. Liang, S. Xu et al., "The use of Au@SiO2 shellisolated nanoparticle-enhanced Raman spectroscopy for human breast cancer detection," Analytical and Bioanalytical Chemistry, vol. 406, no. 22, pp. 5425-5432, 2014.

[26] B. Brozek-Pluska, J. Musial, R. Kordek, E. Bailo, T. Dieing, and H. Abramczyk, "Raman spectroscopy and imaging: applications in human breast cancer diagnosis," The Analyst, vol. 137, no. 16, pp. 3773-3780, 2012.

[27] . Emerman, S. J. Burwen, and D. R. Pitelka, "Substrate properties influencing ultrastructural differentiation of mammary 
epithelial cells in culture," Tissue \& Cell, vol. 11, no. 1, pp. 109$119,1979$.

[28] M. J. Bissell, D. C. Radisky, A. Rizki, V. M. Weaver, and O. W. Petersen, "The organizing principle: microenvironmental influences in the normal and malignant breast," Differentiation; Research in Biological Diversity, vol. 70, no. 9-10, pp. 537-546, 2002.

[29] N. P. Damayanti, Y. Fang, M. R. Parikh, A. P. Craig, J. Kirshner, and J. Irudayaraj, "Differentiation of cancer cells in twodimensional and three-dimensional breast cancer models by Raman spectroscopy," Journal of Biomedical Optics, vol. 18, no. 11, p. 117008, 2013.

[30] M. P. Shekhar, R. Pauley, and G. Heppner, "Host microenvironment in breast cancer development: extracellular matrixstromal cell contribution to neoplastic phenotype of epithelial cells in the breast," Breast Cancer Research: BCR, vol. 5, no. 3, pp. 130-135, 2003.

[31] A. S. Haka, Z. Volynskaya, J. A. Gardecki et al., "In vivo margin assessment during partial mastectomy breast surgery using raman spectroscopy," Cancer Research, vol. 66, no. 6, pp. 3317-3322, 2006.

[32] M. D. Keller, S. K. Majumder, and A. Mahadevan-Jansen, "Spatially offset Raman spectroscopy of layered soft tissues," Optics Letters, vol. 34, no. 7, pp. 926-928, 2009.

[33] M. D. Keller, E. Vargis, N. de Matos Granja et al., “Development of a spatially offset Raman spectroscopy probe for breast tumor surgical margin evaluation," Journal of Biomedical Optics, vol. 16, no. 7, p. 077006, 2011.

[34] A. M. Mohs, M. C. Mancini, S. Singhal et al., "Hand-held spectroscopic device for in vivo and intraoperative tumor detection: contrast enhancement, detection sensitivity, and tissue penetration," Analytical Chemistry, vol. 82, no. 21, pp. 90589065, 2010.

[35] M. Gipponi, C. Bassetti, G. Canavese et al., "Sentinel lymph node as a new marker for therapeutic planning in breast cancer patients," Journal of Surgical Oncology, vol. 85, no. 3, pp. 102111, 2004.

[36] J. Wong, W. S. Yong, A. A. Thike et al., "False negative rate for intraoperative sentinel lymph node frozen section in patients with breast cancer: a retrospective analysis of patients in a single Asian institution," Journal of Clinical Pathology, vol. 68, no. 7, pp. 536-540, 2015.

[37] M. Isabelle, N. Stone, H. Barr, M. Vipond, N. Shepherd, and K. Rogers, "Lymph node pathology using optical spectroscopy in cancer diagnostics," Spectrometry International Journal, vol. 22, no. 2-3, pp. 97-104, 2008.

[38] J. Smith, C. Kendall, A. Sammon, J. Christie-Brown, and N. Stone, "Raman spectral mapping in the assessment of axillary lymph nodes in breast cancer," Technology in Cancer Research \& Treatment, vol. 2, no. 4, pp. 327-332, 2003.

[39] J. Horsnell, P. Stonelake, J. Christie-Brown et al., "Raman spectroscopy-a new method for the intra-operative assessment of axillary lymph nodes," The Analyst, vol. 135, no. 12, pp. 30423047, 2010.

[40] J. D. Horsnell, J. A. Smith, M. Sattlecker et al., "Raman spectroscopy-a potential new method for the intra-operative assessment of axillary lymph nodes," The Surgeon: Journal of the Royal Colleges of Surgeons of Edinburgh and Ireland, vol. 10, no. 3, pp. 123-127, 2012.

[41] U. Neugebauer, T. Bocklitz, J. H. Clement, C. Krafft, and J. Popp, "Towards detection and identification of circulating tumour cells using Raman spectroscopy," The Analyst, vol. 135, no. 12, pp. 3178-3182, 2010.

[42] S. Dochow, C. Krafft, U. Neugebauer et al., "Tumour cell identification by means of Raman spectroscopy in combination with optical traps and microfluidic environments," Lab on a Chip, vol. 11, no. 8, pp. 1484-1490, 2011.

[43] S. Dochow, C. Beleites, T. Henkel et al., "Quartz microfluidic chip for tumour cell identification by Raman spectroscopy in combination with optical traps," Analytical and Bioanalytical Chemistry, vol. 405, no. 8, pp. 2743-2746, 2013.

[44] M. K. Hossain, H. Y. Cho, K. J. Kim, and J. W. Choi, "In situ monitoring of doxorubicin release from biohybrid nanoparticles modified with antibody and cell-penetrating peptides in breast cancer cells using surface-enhanced Raman spectroscopy," Biosensors \& Bioelectronics, vol. 71, pp. 300-305, 2015.

[45] X. Wan, X. Zheng, X. Pang, Z. Zhang, and Q. Zhang, "Incorporation of lapatinib into human serum albumin nanoparticles with enhanced anti-tumor effects in HER2-positive breast cancer," Colloids and Surfaces. B, Biointerfaces, vol. 136, pp. 817827, 2015.

[46] F. Hu, Y. Zhang, G. Chen, C. Li, and Q. Wang, "Double-walled $\mathrm{Au}$ nanocage/SiO2 nanorattles: integrating SERS imaging, drug delivery and photothermal therapy," Small, vol. 11, pp. 985-993, 2015.

[47] J. A. Menendez, "Fine-tuning the lipogenic/lipolytic balance to optimize the metabolic requirements of cancer cell growth: molecular mechanisms and therapeutic perspectives," Biochimica et Biophysica Acta, vol. 1801, no. 3, pp. 381-391, 2010.

[48] X. Bi, B. Rexer, C. L. Arteaga, M. Guo, and A. Mahadevan-Jansen, "Evaluating HER2 amplification status and acquired drug resistance in breast cancer cells using Raman spectroscopy," Journal of Biomedical Optics, vol. 19, no. 2, p. 025001, 2014.

[49] H. Ilkhani, T. Hughes, J. Li, C. J. Zhong, and M. Hepel, "Nanostructured SERS-electrochemical biosensors for testing of anticancer drug interactions with DNA," Biosensors \& Bioelectronics, vol. 80, pp. 257-264, 2016.

[50] U. S. Dinish, C. Y. Fu, K. S. Soh, B. Ramaswamy, A. Kumar, and M. Olivo, "Highly sensitive SERS detection of cancer proteins in low sample volume using hollow core photonic crystal fiber," Biosensors \& Bioelectronics, vol. 33, no. 1, pp. 293-298, 2012.

[51] A. Nilstad, T. E. Andersen, E. Kristianslund et al., "Physiotherapists can identify female football players with high knee valgus angles during vertical drop jumps using real-time observational screening," The Journal of Orthopaedic and Sports Physical Therapy, vol. 44, no. 5, pp. 358-365, 2014.

[52] S. Lee, H. Chon, J. Lee et al., "Rapid and sensitive phenotypic marker detection on breast cancer cells using surfaceenhanced Raman scattering (SERS) imaging," Biosensors \& Bioelectronics, vol. 51, pp. 238-243, 2014.

[53] M. B. Wabuyele, F. Yan, and T. Vo-Dinh, "Plasmonics nanoprobes: detection of single-nucleotide polymorphisms in the breast cancer BRCA1 gene," Analytical and Bioanalytical Chemistry, vol. 398, no. 2, pp. 729-736, 2010.

[54] H. Hwang, H. Chon, J. Choo, and J. K. Park, "Optoelectrofluidic sandwich immunoassays for detection of human tumor marker using surface-enhanced Raman scattering," Analytical Chemistry, vol. 82, no. 18, pp. 7603-7610, 2010.

[55] S.Lee,H.Chon,S.Y.Yoonetal.,"FabricationofSERS-fluorescence dual modal nanoprobes and application to multiplex cancer cell imaging," Nanoscale, vol. 4, no. 1,pp. 124-129, 2012. 
[56] S. Kang, Y. Wang, N. P. Reder, and J. T. Liu, "Multiplexed molecular imaging of biomarker-targeted SERS nanoparticles on fresh tissue specimens with channel-compressed spectrometry," PloS One, vol. 11, no. 9, Article ID e0163473, 2016.

[57] M. Tonegutti and V. Girardi, "Stereotactic vacuum-assisted breast biopsy in 268 nonpalpable lesions," La Radiologia Medica, vol. 113, no. 1, pp. 65-75, 2008.

[58] R. J. Jackman and J. Rodriguez-Soto, "Breast microcalcifications: retrieval failure at prone stereotactic core and vacuum breast biopsy-frequency, causes, and outcome," Radiology, vol. 239, no. 1, pp. 61-70, 2006.

[59] I. Barman, N. C. Dingari, A. Saha et al., "Application of Raman spectroscopy to identify microcalcifications and underlying breast lesions at stereotactic core needle biopsy," Cancer Research, vol. 73, no. 11, pp. 3206-3215, 2013.

[60] A. Saha, I. Barman, N. C. Dingari et al., "Raman spectroscopy: a real-time tool for identifying microcalcifications during stereotactic breast core needle biopsies," Biomedical Optics Express, vol. 2, no. 10, pp. 2792-2803, 2011.

[61] M. P. Morgan, M. M. Cooke, and G. M. McCarthy, "Microcalcifications associated with breast cancer: an epiphenomenon or biologically significant feature of selected tumors?" Journal of Mammary Gland Biology and Neoplasia, vol. 10, no. 2, pp. 181-187, 2005.

[62] P. Matousek and N. Stone, "Emerging concepts in deep Raman spectroscopy of biological tissue," The Analyst, vol. 134, no. 6, pp. 1058-1066, 2009.

[63] R. Baker, K. D. Rogers, N. Shepherd, and N. Stone, "New relationships between breast microcalcifications and cancer," British Journal of Cancer, vol. 103, no. 7, pp. 1034-1039, 2010.

[64] L. Liang, C. Zheng, H. Zhang et al., "Exploring type II microcalcifications in benign and premalignant breast lesions by shell-isolated nanoparticle-enhanced Raman spectroscopy (SHINERS), Spectrochimica acta," Part a, Molecular and Biomolecular Spectroscopy, vol. 132, pp. 397-402, 2014.

[65] P. Toniolo, A. L. Van Kappel, A. Akhmedkhanov et al., "Serum carotenoids and breast cancer," American Journal of Epidemiology, vol. 153, no. 12, pp. 1142-1147, 2001.

[66] G. E. Dressing, T. P. Knutson, M. J. Schiewer et al., "Progesterone receptor-cyclin D1 complexes induce cell cycle-dependent transcriptional programs in breast cancer cells," Molecular Endocrinology, vol. 28, no. 4, pp. 442-457, 2014.

[67] M. C. Potcoava, G. L. Futia, J. Aughenbaugh, I. R. Schlaepfer, and E. A. Gibson, "Raman and coherent anti-Stokes Raman scattering microscopy studies of changes in lipid content and composition in hormone-treated breast and prostate cancer cells," Journal of Biomedical Optics, vol. 19, no. 11, p. 111605, 2014.

[68] M. Hedegaard, C. Krafft, H. J. Ditzel, L. E. Johansen, S. Hassing, and J. Popp, "Discriminating isogenic cancer cells and identifying altered unsaturated fatty acid content as associated with metastasis status, using $\mathrm{k}$-means clustering and partial least squares-discriminant analysis of Raman maps," Analytical Chemistry, vol. 82, no. 7, pp. 2797-2802, 2010. 

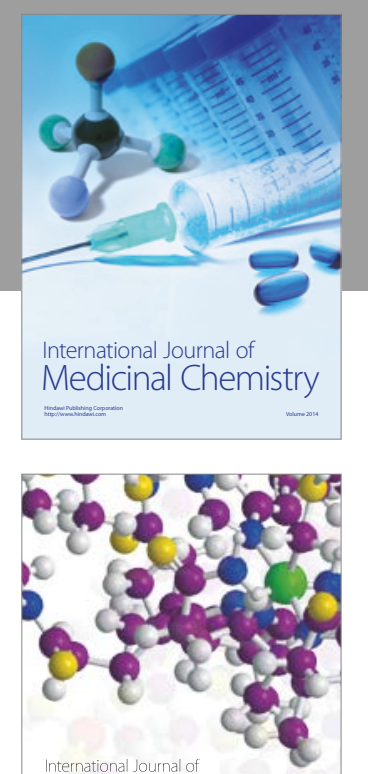

Carbohydrate Chemistry

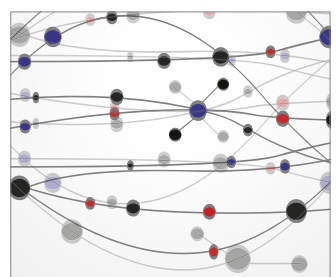

The Scientific World Journal
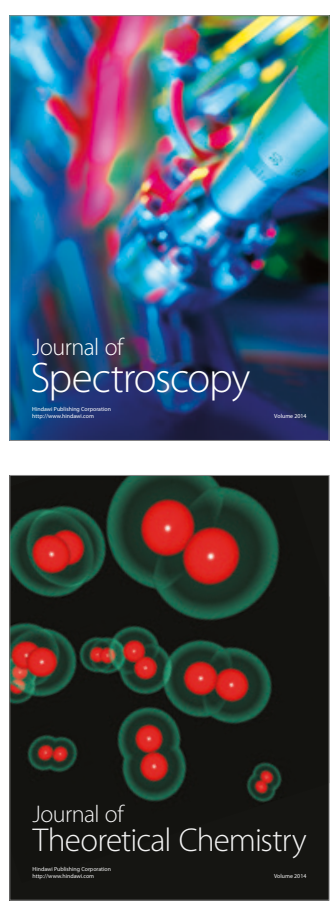
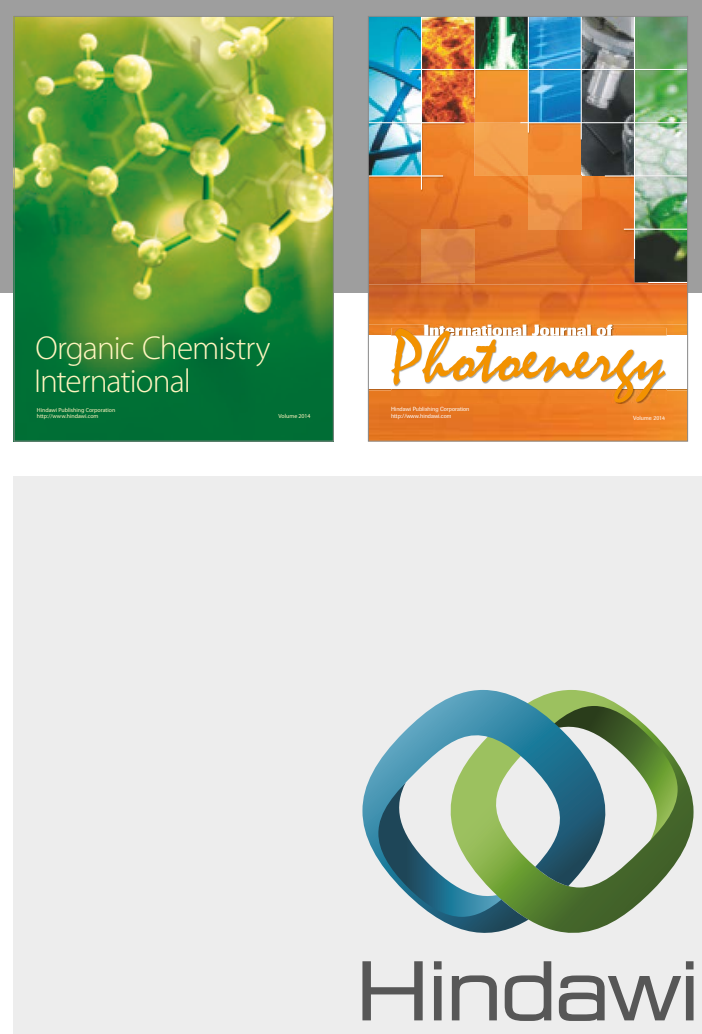

Submit your manuscripts at

https://www.hindawi.com

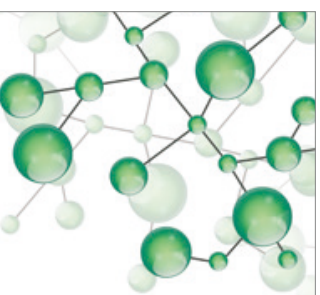

International Journal of

Inorganic Chemistry

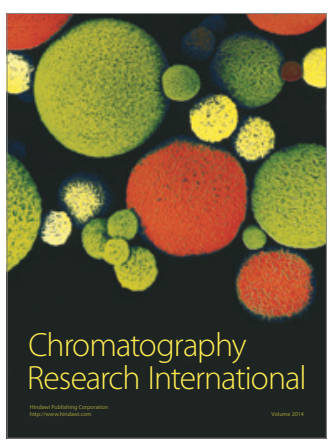

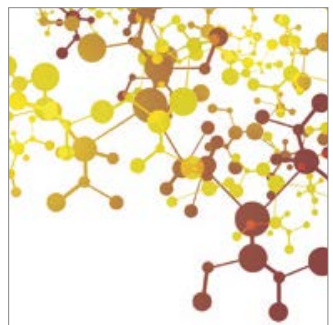

Applied Chemistry
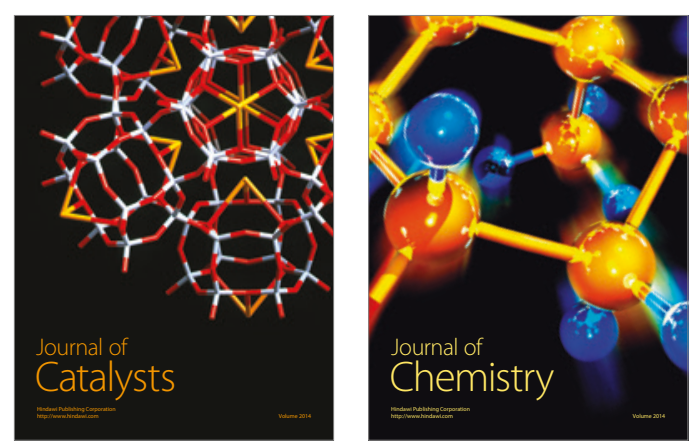
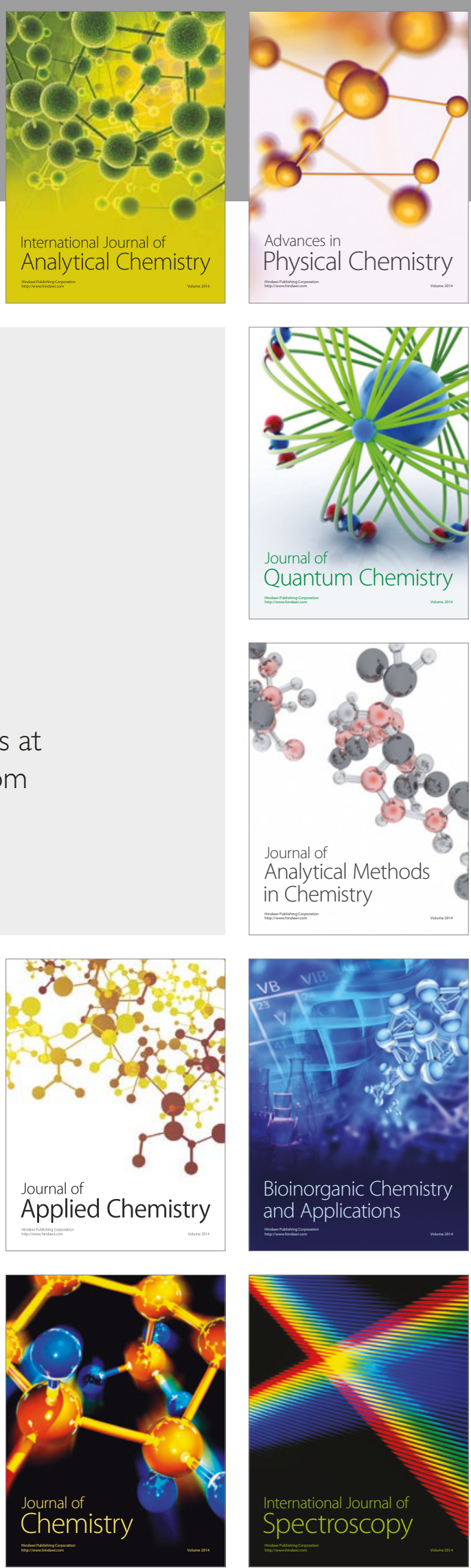